\title{
Enabling IoT Platform Interoperability Using a Systematic Development Approach By Example
}

\author{
Michael Schneider, Benjamin Hippchen, Sebastian Abeck \\ Research Group Cooperation \& Management \\ Karlsruhe Institute of Technology (KIT) \\ Zirkel 2, 76131 Karlsruhe, Germany \\ (michael.schneider | benjamin.hippchen | abeck)@kit.edu
}

\author{
Michael Jacoby, Reinnhard Herzog \\ Fraunhofer IOSB \\ Fraunhoferstr. 1, 76131 Karlsruhe, Germany \\ (michael.jacoby | reinhard.herzog)@iosb.fraunhofer.de
}

\begin{abstract}
Today, the IoT landscape consists of a large number of vertical IoT platforms that are rarely interconnected. To enable creation of applications across platform and domain boundaries interoperability needs to be established between IoT platforms. As this is a challenging task, we present in this paper how to simplify it by utilizing a systematic software development process based on behavior- and domain-driven development. Additionally, we illustrate this process using an example of two indoor navigation platforms based on BLE beacons and the open source IoT interoperability framework symbIoTe. We show that developers can actually profit from this approach but existing IoT interoperability frameworks are still cumbersome to use.
\end{abstract}

Keywords-Internet of Things, IoT, interoperability, semantic interoperability, behavior-driven development, domain-driven design

\section{INTRODUCTION}

The landscape of Internet of Things (IoT) platforms is constantly growing. Most of these platforms are highly specialized on a specific domain or even an application. This results in the IoT landscape being fragmented into many vertical IoT silos which are rarely interconnected. However, the key feature of the IoT is to interconnect things regardless of the way they are physically connected which means that interoperability should not only be possible within platforms but also across platforms.

To enable interoperability across IoT platforms multiple levels of interoperability need to be addressed. The two most important ones are technical interoperability and semantic interoperability. Technical interoperability enables the exchange of data. This is well addressed by existing communication protocols and standards. Thus, it can be treated as more or less solved. However, exchanging data is not enough as we also need to understand it. The ability to understand exchanged data is referred to as semantic interoperability. Enabling semantic interoperability can be far more complex than technical interoperability and is still an active area of research.

In this paper we present by example how to utilize software development approaches and processes to simplify establishing interoperability between two existing IoT platforms. Therefore we will use behavior-driven development (BDD) and domaindriven design (DDD) together with the open source IoT interoperability framework symbIoTe.
The remainder of this paper is structured as follows. Section II provides background information on BDD, DDD and the used symbIoTe framework as well as related work on other IoT interoperability approaches. Furthermore, the example used throughout the paper is introduced in this section. Section III and Section IV present how the software development approaches and processes are applied to the example in the analysis and the design phase. In Section V is shown how (semantic) interoperability is implemented for our example using the symbIoTe interoperability framework. The paper closes with conclusions in Section VI.

\section{BACKGROUND \& RELATED WORK}

\section{A. Software Development Approaches}

The development process, which we apply in our case study is based on behavior-driven development (BDD) and domaindriven design (DDD). Each of the two approaches covers a different aspect of the application. One first step to merge both approaches is given in [1]. The authors classify them into a widely accepted software engineering approach from Brügge et al. [2].

BDD could be seen as a further development of testdriven development (TDD) [3]. TDD tries to determine the correctness of an application with executable acceptance tests, which are written in the chosen programming language [4]. This is the first difference to BDD, which specifies the acceptance tests in the business readable, domain specific language Gherkin[3]. Gherkin uses the common speech and predefined keywords for defining its features. This allows practitioners of BDD to create features directly with the customer, as they are "understood by everyone" [3]. Further, the philosophy of BDD is progressing the application from the outside to the inside [3]. The most visible functionality is first specified as a feature and then implemented directly. During the implementation, new functions are discovered, defined as features and implemented. North characterizes this approach as "code-by-example" [5].

According to Evans, the main reason why applications do not meet the customer's expectations and needs is the lack of knowledge from the developers of the customer's domain [6]. To address this problem, Evans introduced the DDD approach with various patterns and principals in his book "Domain-Driven Design: Tackling Complexity in the Hearth of 
Software". With DDD, the customer's domain is analysed and the results-or the so-called domain knowledge-are stated in a domain model. By establishing a "ubiquitous language", the development team and the customer speak the same language. An essential principal of DDD is, that the source code of the application reflects the structure of the domain model.

\section{B. symbIoTe}

symbIoTe (symbiosis of smart objects across IoT environments) [7] is an $\mathrm{H} 2020$ EU project. Its main objective is to provide an open source interoperability and mediation framework for collaboration and federation of vertical IoT platforms. symbIoTe provides interoperability on four so-called interoperability levels (L1-L4): syntactic and semantic interoperability (L1), platform federations (L2), dynamic smart spaces (L3) and roaming devices (L4). In our example, we are going to make the platforms only L1-compliant as L1 already covers our needs (syntactic and semantic interoperability).

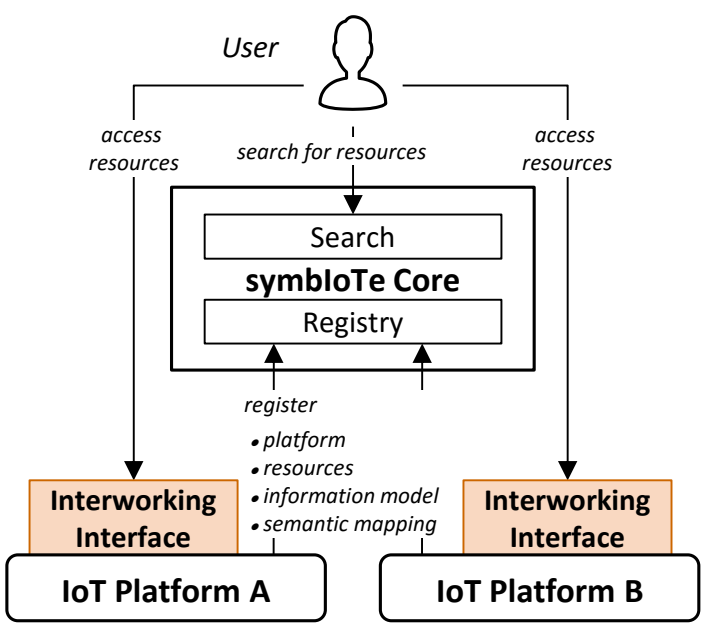

Fig. 1. High-level overview of symbIoTe L1 functionality.

Figure 1 shows a high-level overview of symbIoTe's L1 functionality. To be symbIoTe L1-compliant, a platform must implement the Interworking Interface. This interface comprises different functionality, most importantly resource access, authentication and authorization. Besides implementing the Interworking Interface, a platform must register to a symbIoTe Core instance providing information about the platform, for example metadata about the platform (e.g. name, owner, URL of Interworking Interface implementation) and its exposed resources. Furthermore, a platform must provide its used information model to the symbIoTe Core (further referred to as Core) upon registration optionally together with semantic mappings (further referred to as mappings) between its model and other models.

The Core is the main entry point for software that uses symbIoTe and provides a search functionality across all the registered resources. The user depicted in Figure 1 does not refer to an end user of an IoT app but rather to a use of the symbIoTe framework, which can be e.g. mobile apps, websites, IoT platforms, backend services, etc. Once a user has discovered a resource he can access it through the Interworking Interface implementation of the platform the resource belongs to which will return him the actual data. This way, the platforms maintain complete control over their data as the Core only sees the meta data used for registration and search but never the actual sensor/actuator data.

In the following, we will present in a bit more detail why this is the case and how exactly syntactic and semantic interoperability are established.

In symbIoTe, syntactic and semantic interoperability are partially interlinked. There are multiple possible approaches to enable semantic interoperability between multiple platfor$\mathrm{m}$ using different information models as presented in [8]. However, they are all trade-offs between two main contradicting approaches which can be called standardization or agreement, meaning that all platforms agree on a common information model and expose their data according to this standardized model, and mapping, meaning that each platform uses whatever information model it likes and interoperability is only established through semantic mapping between the models. symbIoTe uses an intermediate approach called Core Information Model with Extensions. In this approach, each platform exposes its data based on a Platform-Specific Information Model (PIM). However, this PIM has to be an extension of the Core Information Model (CIM), which is a generic and abstract model shared between all platforms. This way symbIoTe provides some interoperability between all platforms out-of-the-box (in terms of the CIM) while supporting any kind of platform-specific extensions. Semantic Interoperability between different PIMs can be established through semantic mappings.

Syntactic interoperability in symbIoTe is realized by exposing the resources of a platform through a REST-based interface. However, as stated above, syntactic and semantic interoperability are interlinked. This is because the URLs exposed for resource access are not statically defined, i.e. the same for every platform, but depend on the PIM of the platform. This idea of an information model-agnostic RESTbased interface is the fundamental concept of the OData protocol ${ }^{1}$ which is why a part of the Interworking Interface dealing with resource access was designed to be very close to the OData protocol.

Looking again at Figure 1, we now understand why a platform must provide its used information model (PIM) to the symbIoTe Core upon registration.

To enable interoperability between two different PIMs, symbIoTe makes use of the semantic mapping information in different ways. In the Core, mappings are utilized to perform query re-writing/translation from one PIM to another, thereby allowing to find resources of platforms using different PIMs with one query. Furthermore, symbIoTe provides a client library that utilizes mappings for data translation, which allows accessing data from another platforms in a PIM-transparent

\footnotetext{
${ }^{1}$ http://www.odata.org/
} 
way. This means, that one platform can access another platform as if it would use the same PIM as long as there is mapping between both PIMs registered in the Core.

Semantic mapping is a very difficult problem and still an open research area. symbIoTe provides a prototype implementation with basic capabilities for this. However, this will be enough for basic scenarios as in our example.

\section{Other IoT interoperability frameworks}

There are a lot of research groups and standards addressing the problem of cross-platform interoperability in the IoT context. These are for example the IRTF Thing-to-Thing Resource Group (T2TRG), W3C Web of Things Working Group (WoT), SensorThingsAPI from the Open Geospatial Consortium (OGC), iot-schema.org, oneM2M and many more. However, these are more fundamental activities whereas we will focus on more hands-on project that are either available right now or are expected to be available soon.

Therefore, in this section we will present three projects which are part of the IoT-European Platforms Initiative (IoT-EPI), all addressing the problem of IoT platform interoperability. IoT-EPI is a European initiative bringing together seven EU-funded research and innovation projects (including symbIoTe) in the area of IoT platform development.

\section{BIG IoT}

Main objective of the BIG IoT project [9] (Bridging the Interoperability Gap of the IoT) is to create an open marketplace for IoT platforms and services. The marketplace concept of BIG IoT is very similar to the symbIoTe Core and also the whole processing of making a platform (L1-) compliant to the system as well as registering and searching resources. BIG IoT uses the W3C WoT ThingDescription to semantically describe resources and allows the usage of any information model. However, they do not provide any support to enable interoperability between platforms using different information models.

\section{bIoTope}

The bIoTope project [10] (Building an IoT Open Innovation Ecosystem For Connected Smart Objects) addresses interoperability with a Systems-of-Systems approach. Everything (e.g. apps, devices, platforms, gateways, non-IoT application and services) has to have a wrapper that exposes the resource's data through the Open Message Interface (O-MI) using the Open Data Format (O-DF). Just like BIG IoT, bIoTope does allow the use of any information model but does not provide any tool support for establishing semantic interoperability.

\section{INTER-IoT}

The INTER-IoT project [11] (Interoperability of heterogeneous IoT platforms) addresses interoperability on five levels; device, network, middleware, application and data, and semantics. Besides symbIoTe, INTER-IoT is the only project in the IoT-EPI explicitly addressing semantic mapping and providing a tool set to make use of these mappings. However, in INTER-IoT, semantic mapping is not done directly between two different platform-specific information models but always from a platform-specific information model to a common, shared model [12].

\section{Introduction of the Example}

Figure 2 shows a schematic representation of the components and their deployment of the running example. The initial position is, that there are two existing IoT platforms, Platform Campus A and Platform Campus B, one deployed at the KIT, the other at Fraunhofer IOSB premises and both providing a a mobile app supporting their offered services. The goal is to enable interoperability between the platform so that users of Campus A can use their existing application (e.g. for searching a room) for the same intention when visiting Campus B. In this case, the application of Campus A requires data (including IoT data, such as the current location of the user) of Campus B. However, as the two platforms may use different information models (called PIMs in symbIoTe) the exchanged data might not be understood by the application. Therefore, the data needs to be provided in a way so that the corresponding application can process the data. A transformation of the information model (like schematic mapping) is needed. This transformation is done by a component of the adapter, as shown in Figure 2. To establish the connection to symbIoTe, Campus A and Campus B have to implement the symbIoTe adapter. For accessing resources from Campus B, the resource access proxy (RAP) of Campus B must be known. This adapter offers the required data from Campus B. Therefore, the adapter registers the platform and provides the information model in the so-called resource description format (RDF) [13] to symbIoTe Core. Afterwards, the resources from Campus B can be searched and found in the Core and are accessible via the interworking interface. The Interworking Interface is RESTbased [14]. The backend of Campus B provides the needed data through a plug-in that communicates with the RAP. The data that Campus A requested from Campus B is received by the symbIoTe client (which is part of the adapter) of Campus A. In this stage, the received data is semantically mapped for the needs of the backend that Campus A provides for their application. On the left side, Campus A has to implement the necessary adapter for enabling symbIoTe. On the right side, Campus B also needs a connection to symbIoTe and has to implement an adapter as well. One challenge of the semantic mapping is that the domains of Campus $\mathrm{A}$ and Campus $\mathrm{B}$ are not the same. Campus A provides a beacon-based navigation service while Campus B offers a room reservation service. The consequence is that the information models are not the same. However, there are several overlaps like determining the current location that can be utilized for the application.

\section{ANALYSIS PHASE: FEATURE DESCRIPTION}

As a member of Campus A, I want to use my application to show my current location on Campus B. This is an important requirement in terms of the interoperability that the desired 


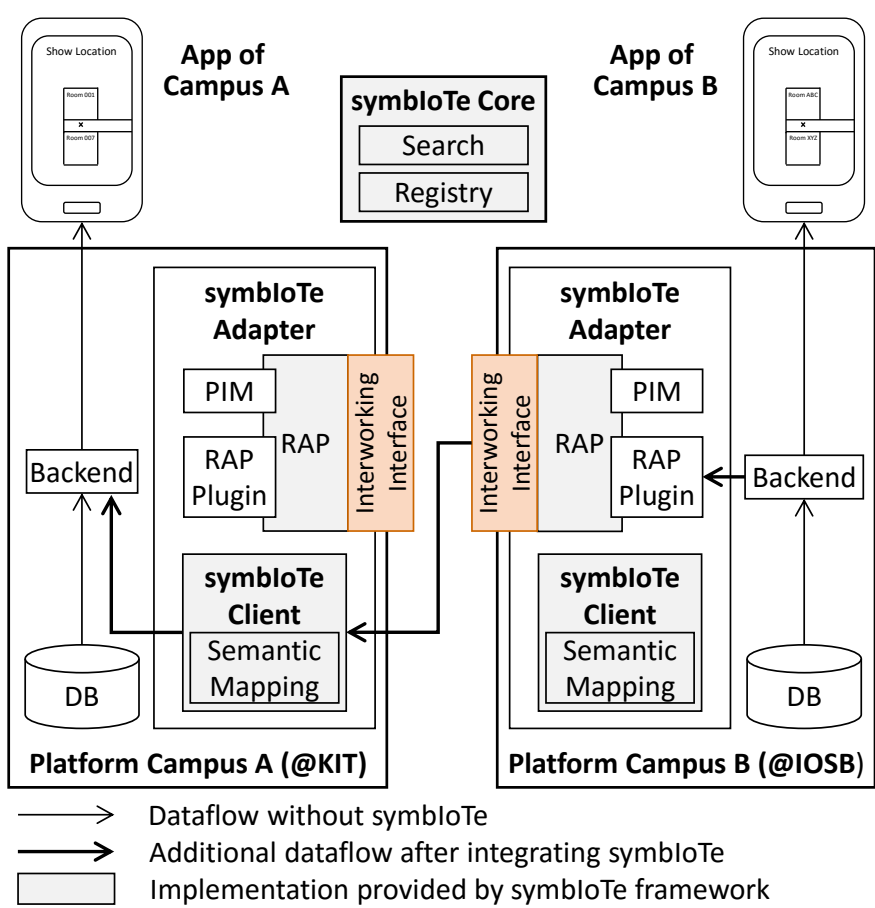

Fig. 2. Schematic representation of components and their deployment for the example use case including the data flow with and without symbIoTe.

application has to fulfil. In order to create a cross-domain application, it is necessary to discuss the need of the interoperability as a requirement. To address this need, behaviordriven development is used for analysing and specifying the requirements. Therefore, the requirements are specified as features. This is the first part of the systematic development process. Figure 3 shows the feature that describes the desired goal of the application. A BDD template is used to specify

1. Feature: Show my location on Campus B

2. As a member of Campus $A$

3. I want to use my well-known application

4. In order to determine my current location on Campus B

5. Scenario: Show my location on Campus B

6. Given I am at Campus B

7. And a beacon from Campus B is available

8. When I open the "Current Location" page

9. Then my current location on Campus B should be displayed

Fig. 3. Campus Interoperability Feature

the feature shown in Figure 3. It consists of two parts; a feature description part [15], that describes the business value of the feature and a scenario description part [3], which is written in natural language. An advantage of the scenarios is that developers can easily understand and discuss the specified features with the stakeholders. The scenarios describe how the application works. In addition, scenarios describe the acceptance criteria of a feature that allows the automated testing of the requirement. In order to test the feature shown in Figure 4, the step definitions have to be implemented.
Before the written tests can pass, the functionality has to be implemented. To be able to test the feature shown in Figure 3 , there must be a connection to symbIoTe. Line 7 of the scenario given in Figure 3 makes it clear that a beacon from Campus B must be available. This step delivers an important hint. Without a connection to symbIoTe, the test cannot pass because the needed data cannot be acquired from Campus B. Thus, the systematic development approach delivers important clues for the developers in terms of interoperability and the developer knows what needs to be implemented. Features also contain scenarios that cover errors that may occur. Therefore, the features provide important clues for the developers in order to allow cross-domain communication. To test a feature even further, each feature contains more than one scenario. The set of scenarios to a feature should also contain scenarios that describe what happens in an error situation.

In addition to the features which concern the cross-domain integration, there is also a need for features that describe the functionality of the application. These features are specified like the features concerning interoperability. Figure 4 shows

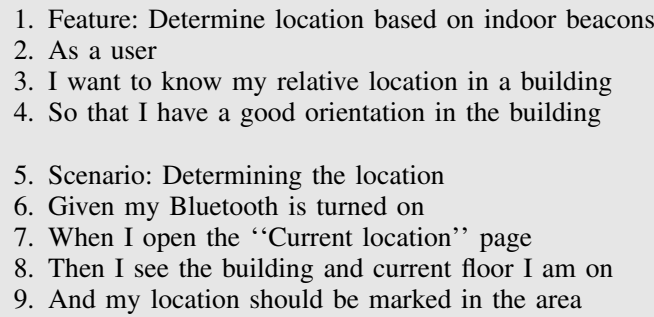

1. Feature: Determine location based on indoor beacons

2. As a user

3. I want to know my relative location in a building

4. So that I have a good orientation in the building

5. Scenario: Determining the location

6. Given my Bluetooth is turned on

7. When I open the "Current location" page

8. Then I see the building and current floor I am on

9. And my location should be marked in the area

10. Scenario: Determining the location with Bluetooth disabled

11. Given my Bluetooth is turned off

12. When I open the "Current location" page

13. Then I should be asked to activate Bluetooth

Fig. 4. Main Feature of NavSG Feature

one of the main features of the indoor navigation application. For the navigation application, it is important to determine the current position of a user. The location of the user is displayed. In addition to the location, further terms like building and floor are used. These terms and other relevant terms are providing domain knowledge and they have influence on the domain model. Therefore, the specified features function as input for the modelling phase.

\section{Design Phase: Modeling and Integration of THE MODELS}

To enable semantic interoperability each platform needs to provide a formally defined model of the domain. In case of Campus A where we applied BDD and DDD, we automatically get this model as part of the systematic development process. For Campus B, the model was created manually based on existing class diagrams. In this section we first introduce the two different models and then analyse their differences and how they can be aligned. Please note, that the models have been simplified to better illustrate the example. 


\section{A. Model of Campus A}

The creation of the domain model from Campus A is based on the features. A feature specifies parts of the business logic from the viewpoint of a user. In addition, each feature specifies parts of the application logic of the software system. A part of the business logic is the domain logic, which is application agnostic. Therefore, a feature contains relevant information about the domain, which is relevant for understanding the domain. The approach to understand the domain and the functionality related to the domain is achieved by reading every line of the feature and its scenarios and identifying the presumably relevant terms. Each relevant term becomes a part of the ubiquitous language, an important concept of DDD [6]. With each analysed feature, additional and relevant terms are identified. For example, the feature shown in Figure 4 led to the terms beacon, location, floor and building. In addition, the relationships between the terms can be derived from the feature, e.g. a beacon is at a location. Further features, knowledge crunching and more insight extended the domain model to the resulting model, as shown in Figure 5.

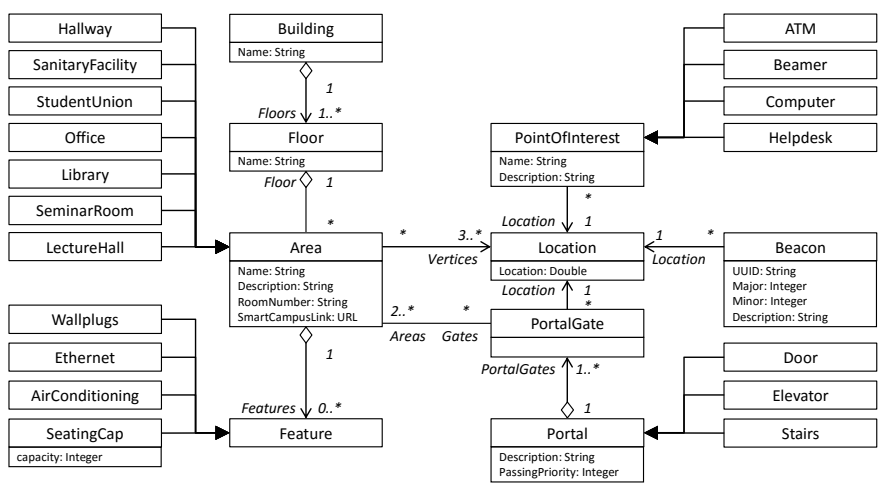

Fig. 5. Domain Model of Campus A

The excerpt from the domain model of the NavSG platform describes the relations of the domain objects. The main concept of the model is the Area. Three or more Locations define the vertices of an Area. Each Floor is divided into several Areas while a Floor is part of a Building. An Area can be a specific type like Hallway or Office. For navigation purposes, each Area has at least one PortalGate at a specific Location. Two or more PortalGates are connected by a Portal. To determine the position of a user, beacons are placed at a specific Location in an Area. For this purpose, it is necessary to distinguish the beacons. Therefore, each beacon has an universally unique identifier (UUID) which is displayed as attribute in the model.

\section{B. Model of Campus B}

Figure 6 depicts the domain model of Campus B using the EduCampus platform. The main concepts are BleBeacons that are attached to a Thing and BeaconDetection which represents events generated when a User was close to a beacon at a certain date and time. Users can also create Reservations for a room and time interval, including Catering Requests. A Thing can be either a Room or a MoveableThing, e.g. inventory. A Room has the properties capacity and roomNo, can have multiple Features like a projector or a whiteboard and can contain multiple Workspaces.

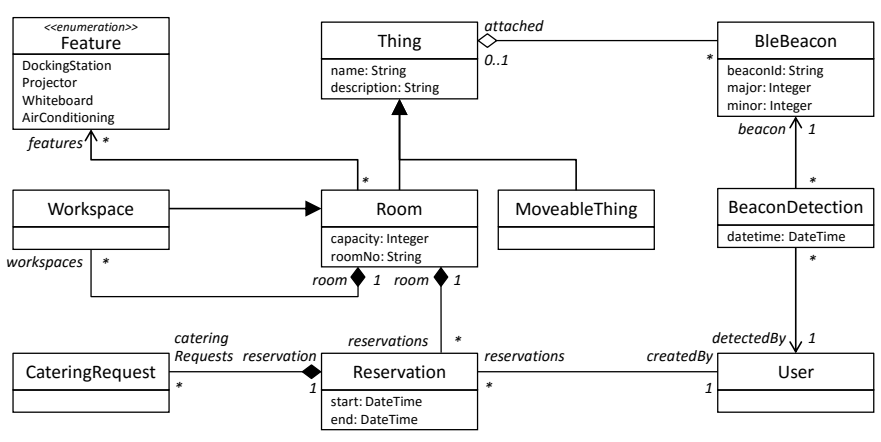

Fig. 6. Domain Model of Campus B.

\section{Differences and Integration of the models}

Although both models cover more or less the same domain, they have quite different views of it. This is caused by different needs of the existing applications resulting in different levels of detail of the model and by design decisions like using ray tracing or nearest-neighbour for location. However, they both provide information about areas/rooms and the functionality to identify the position of a user based on the beacons in range. To share this functionality between the platforms, both expose a service called getPosition taking some beacon information as input and returning the position. However, they use the corresponding classes of their domain model to describe the input and return type. Therefore, the service definition for Campus A is getPosition(Beacon[]) $\rightarrow$ Area and for Campus B getPosition(BleBeacon[]) $\rightarrow$ Room. This reduces the problem of semantic interoperability to mapping the concepts Area and Room as well as Beacon and BleBeacon. This mapping has to include all the properties and references of these concepts that are present in both models.

Mapping Beacon and BleBeacon is straightforward by renaming the shared properties (UUID $\leftrightarrow$ beaconId, Major $\leftrightarrow$ major, Minor $\leftrightarrow$ minor) and dropping the optional property Description. To map Area and Room we again start with renaming the shared properties Name $\leftrightarrow$ name, Description $\leftrightarrow$ description and RoomNumber $\leftrightarrow$ roomNo. The rest of the mapping, covering features of an area/room and the seating capacity, is more complex as it involves relations between objects. If an Area has a Feature of type SeatingCap we map its property capacity to the property capacity of Room and the other way round. Further, we map the only shared type of feature from an instance in the model of Campus A to the corresponding enumeration value in the model of Campus B.

This mapping needs to be formally defined to be used with symbIoTe. At the moment of writing, semantic mapping functionality of symbIoTe is still work in progress ${ }^{2}$ and therefore we cannot provide such a formal definition. However, the

\footnotetext{
2 https://github.com/symbiote-h2020/SemanticMapping
} 
language used to express such a semantic mapping will be very close to the Expressive and Declarative Ontology Alignment Language [16] (EDOAL) and most likely compatible to it.

\section{Implementation Phase: Current State}

The systematic development process is an agile approach which means that the implementation of the software should start as soon as possible. Implementation of the domain model is an early activity of the implementation phase, which is carried out before any architectural design is specified. In addition to the domain model, the step definitions of the features which were specified in the analysis phase are implemented and the technology-independent, microservices-based architecture [17] is specified. The specification of the API depends on the domain objects of the domain model that should be exposed. The implementation of the frontend and backend parts are based on the technology-independent API specification. In addition, the adapter required by symbIoTe is implemented. Therefore, the specified API is close to the domain model and is used for the data exchange. At this point, the implementation of the semantic mapping component is still in progress; thus the API will be available soon. For registering with the core, an ontology is extracted from the domain model and provides the domain model in the RDF format. All implementation work is carried out in a test-driven manner based on unit tests and user acceptance tests which are defined by the scenarios of the features.

\section{CONCLUSION}

In this paper we have presented a real-life example of how to establish interoperability between two IoT platforms dealing with BLE-based indoor localization. We used symbIoTe as an open source IoT interoperability framework and showed that using a systematic software development based on BDD and DDD is helpful in this process. This is because semantic interoperability is a very tough problem (besides when standardization is used) and BDD and DDD implicitly output a domain model, which is needed to achieve semantic interoperability. It is the authors' opinion that utilizing/identifying/creating systematic software development processes can significantly reduce the complexity of enabling interoperability between IoT platforms, especially regarding semantic interoperability.

IoT interoperability is a growing area of research and will even grow faster in the future as the number of internet connected devices keeps increasing. Even though it is currently addressed by multiple research projects as described in Section II-C, further research and standardization is needed to provide easy-to-use interoperability libraries and frameworks to be used by non-expert programmers.

\section{ACKNOWLEDGMENT}

This work is supported by the H2020 symbIoTe project, which has received funding from the European Union's Horizon 2020 research and innovation programme under grant agreement No 688156.

\section{REFERENCES}

[1] B. Hippchen, P. Giessler, R. Steinegger, M. Schneider, and S. Abeck, "Designing microservice-based applications by using a domain-driven design approach," in International Journal on Advances in Software. IARIA, 2017, pp. 432-445.

[2] B. Bruegge and A. H. Dutoit, Object-Oriented Software Engineering Using UML, Patterns and Java-(Required). Prentice Hall, 2004.

[3] M. Wynne, A. Hellesoy, and S. Tooke, The cucumber book: behaviourdriven development for testers and developers. Pragmatic Bookshelf, 2017.

[4] K. Beck, Test-Driven Development: By Example. Addison-Wesley Professional, 2003.

[5] D. North. (2009) BDD \& DDD. QCon London 2009. URL: https://www. infoq.com/presentations/bdd-and-ddd [retrieved: 2017.11.30].

[6] E. Evans, Domain-Driven Design: Tackling Complexity in the Heart of Software. Addison-Wesley Professional, 2004.

[7] S. Soursos, I. P. Žarko, P. Zwickl, I. Gojmerac, G. Bianchi, and G. Carrozzo, "Towards the cross-domain interoperability of iot platforms," in 2016 European Conference on Networks and Communications, 2016.

[8] M. Jacoby, A. Antonić, K. Kreiner, R. Łapacz, and J. Pielorz, "Semantic interoperability as key to iot platform federation," in International Workshop on Interoperability and Open-Source Solutions. Springer, 2016, pp. 3-19.

[9] A. Bröring, S. Schmid, C.-K. Schindhelm, A. Khelil, S. Käbisch, D. Kramer, D. Le Phuoc, J. Mitic, D. Anicic, and E. Teniente, "Enabling iot ecosystems through platform interoperability," IEEE software, vol. 34, no. 1, pp. 54-61, 2017.

[10] bIoTope project. [Online]. Available: http://www.biotope-project.eu/

[11] G. Fortino, C. Savaglio, C. E. Palau, J. S. de Puga, M. Ganzha, M. Paprzycki, M. Montesinos, A. Liotta, and M. Llop, "Towards multi-layer interoperability of heterogeneous iot platforms: The interiot approach," in Integration, Interconnection, and Interoperability of IoT Systems. Springer, 2018, pp. 199-232.

[12] M. Ganzha, M. Paprzycki, W. Pawłowski, P. Szmeja, and K. Wasielews$\mathrm{ka}$, "Towards semantic interoperability between internet of things platforms," in Integration, Interconnection, and Interoperability of IoT Systems. Springer, 2018, pp. 103-127.

[13] O. Lassila, R. R. Swick et al., "Resource description framework (rdf) model and syntax specification," 1998.

[14] R. T. Fielding, "Rest: architectural styles and the design of networkbased software architectures," Doctoral dissertation, University of California, 2000.

[15] D. North et al., "What's in a story," 2016. [Online]. Available: https://dannorth.net/whats-in-a-story/

[16] J. Euzenat, "Edoal: Expressive and declarative ontology alignment language," URL: http://alignapi. gforge. inria. fr/edoal. html (visited on 22/08/2015), 2015.

[17] S. Newman, Building Microservices: Designing Fine-grained Systems. " O'Reilly Media, Inc.", 2015. 